\title{
Particionamento de Grafos Cordais em Conjuntos Independentes e Cliques
}

P. HELL ${ }^{1}$, School of Computing Science, Simon Fraser University, Burnaby, B.C., Canada, V5A1S6.

S. KLEIN ${ }^{2}$ IM e COPPE/Sistemas, Universidade Federal do Rio de Janeiro, RJ, 21945-970, Brasil.

L. T. NOGUEIRA ${ }^{3}$, COPPE/Sistemas, Universidade Federal do Rio de Janeiro, RJ, 21945-970, Brasil.

F. PROTTI 4 , NCE, Universidade Federal do Rio de Janeiro, RJ, 20001-970, Brasil.

Resumo. Neste trabalho consideramos a seguinte generalização de grafos split: Um grafo $G$ é um grafo- $(k, l)$ se seu conjunto de vértices pode ser particionado em $k$ conjuntos independentes e $l$ cliques (uma clique é um subgrafo completo não necessariamente maximal). Portanto, os grafos- $(k, l)$ são uma generalização dos grafos split, que correspondem aos grafos- $(1,1)$. Grafos split podem ser eficientemente reconhecidos [4]. Além disso, os problemas clássicos de otimização combinatória são também resolvidos eficientemente nessa classe. Na verdade, nosso resultado principal é uma caracterização de grafos cordais- $(k, l)$. Também apresentamos um algoritmo para reconhecer grafos cordais- $(k, l)$ cuja complexidade é $O(n(m+n))$. Quando $k=l=1$, nosso algoritmo possui complexidade $O(m+n)$.

Em particular, obtivemos um algoritmo mais simples e eficiente apra reconhecer grafos split, do qual torna-se fácil derivar a conhecida caracterização de grafos split por subgrafos proibidos.

\section{Introdução}

Um grafo $G$ é um grafo- $(k, l)[1]$ se seus vértices podem ser particionados em $k$ conjuntos independentes e $l$ cliques. (Uma clique é um subgrafo completo não necessariamente maximal.) Portanto, os grafos- $(k, l)$ são uma generalização dos grafos split [7], que correspondem aos grafos- $(1,1)$. Grafos split podem ser eficientemente

\footnotetext{
${ }^{1}$ pavol@cs.sfu.ca

${ }^{2}$ sula@cos.ufrj.br

${ }^{3}$ loana@cos.ufrj.br

${ }^{4}$ fabiop@cos.ufrj.br
} 
reconhecidos [7]. Além disso, os problemas clássicos de otimização combinatória são também resolvidos eficientemente nessa classe. Quando $k>1$ ou $l>1$, os grafos-( $k, l)$ não são em geral cordais. (Um grafo é dito cordal se não possui $C_{k}$, um ciclo de $k$ vértices sem cordas, para $k \geq 4$, como subgrafo induzido.) Em [2], algoritmos polinomiais de reconhecimento para as classes $(2,1),(1,2)$ e $(2,2)$ foram propostos. Feder et al. [3] também propuseram algoritmos polinomiais de reconhecimento para essas classes, que surgiram como sub-produto de algoritmos de partição em subgrafos densos e esparsos. Por outro lado, sabe-se que reconhecer grafos- $(k, l)$ para $k \geq 3$ ou $l \geq 3$ é $N P$-completo [1]. (Por exemplo, a classe dos grafos- $(k, 0)$ corresponde ao problema de reconhecer se um dado grafo é $k$-colorível.).

Neste trabalho apresentamos uma caracterização para a classe dos grafos cordais$(k, l)$, assim como um algoritmo polinomial para o reconhecimento desta classe de grafos. Mais especificamente, provamos que um grafo cordal é um grafo- $(k, l)$ se, e somente se, não contém $l+1$ cópias independentes de $K_{k+1}$. (Um conjunto de subgrafos é independente se eles são disjuntos e dois a dois não adjacentes, i.e., não ligados por nenhuma aresta. A notação $K_{r}$ designa uma clique com $r$ vértices.)

Um caso especial deste resultado para grafos cordais- $(2,1)$ foi inicialmente tratado em [5].

Uma forma alternativa de enunciar nosso resultado baseia-se no seguinte fato: o número máximo de $K_{r}^{\prime} s$ independentes num grafo cordal é igual ao número mínimo de cliques necessárias para tocar todos os $K_{r}^{\prime} s$ de $G$. Em outras palavras, se denotarmos por $f(G, r)$ o número máximo de cópias independentes de $K_{r}^{\prime} s$ em $G$, e por $g(G, r)$ o número mínimo de cliques que interceptam todos os $K_{r}^{\prime} s$ de $G$, então podemos mostrar que para um grafo cordal $f(G, r)=g(G, r)$. (Observe que quando $r=1, f(G, r)$ é o número de independência de $G$, e $g(G, r)$ é o número de cobertura por cliques de $G$.). Nosso algoritmo de tempo $O(n(m+n))$ identifica $f(G, r)$ cópias independentes de $K_{r}^{\prime} s$ e o mesmo número de cliques que interceptam todos os $K_{r}^{\prime} s$. Nosso algoritmo de reconhecimento na verdade encontra o menor valor de $l$ para o qual $G$ é um grafo- $(k, l)$. O algoritmo se torna mais eficiente quando $k=1$, i.e., quando procuramos uma partição do grafo em um conjunto independente e um conjunto de cliques. Quando ambos $k$ e $l$ são iguais a um, especializamos o algoritmo para gerar um algoritmo mais simples e eficiente para grafos split. (Observe que neste caso nós não precisamos da restrição de grafo cordais.)

Seja $G$ um grafo. Se $S, S^{\prime} \subseteq V(G)$, denotamos por $N_{S}\left(S^{\prime}\right)$ a vizinhança de $S^{\prime}$ em $S$, i.e., o conjunto de vértices de $S$ que estão em $S^{\prime}$ ou que são adjacentes a algum vértice de $S^{\prime}$. Além disso, se $N_{S}\left(S^{\prime}\right) \neq \emptyset$, então nós dizemos que $S$ e $S^{\prime}$ são adjacentes.

Escreveremos $N_{S}(v)$ ao invés de $N_{S}(\{v\})$, observe que esta vizinhança de $v$ em $S$ contém $v$ se $v \in S$.

\section{Teoremas}

Nesta seção apresentamos nossa caracterização de grafos cordais- $(k, l)$ em termos de subgrafos proibidos. Os seguintes lemas serão úteis: 
Lema 1. Sejam $C$ e $C^{\prime}$ duas cliques (adjacentes ou não) num grafo cordal $G$. Então algum vértice de $C^{\prime}$ é adjacente a todos os vértices de $N_{C}\left(C^{\prime}\right)$.

Prova. Devemos provar que, na verdade, os vizinhos de $C^{\prime}$ em $C$ são linearmente ordenados por inclusão. Suponha que dois vértices distintos $v_{1}, v_{2} \in C^{\prime}$ tem vizinhos incomparáveis em $C$, i.e., nenhum dos conjuntos $N_{C}\left(v_{1}\right), N_{C}\left(v_{2}\right)$ contém o outro. Então existem dois vértices distintos $u_{1}, u_{2} \in C$ tal que $u_{1}$ é adjacente a $v_{1}$ mas não o é a $v_{2}$, e $u_{2}$ é adjacente a $v_{2}$ mas não o é a $v_{1}$. Isto é impossível, já que $u_{1}, u_{2}, v_{2}, v_{1}$ induziriam um ciclo de tamanho 4 sem corda, como mostra a Figura 1. O lema segue considerando o vértice $v \in C^{\prime} \operatorname{com} N_{C}(v)$ máximo.

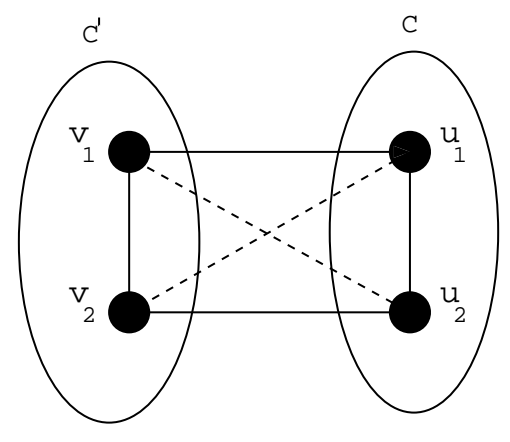

Figura 1: Exemplo para o Lema 1

Lema 2. Sejam $C$ e $K$ duas cliques disjuntas (adjacentes ou não) de um grafo cordal $G$. Então existe uma clique $C^{\prime}$ com a seguinte propriedade: $C^{\prime}$ intercepta $K$, e também intercepta todas as cliques adjacentes a $K$ que são interceptadas por $C$.

Prova. Seja $L=N_{C}(K)$. Pelo Lema 1 algum vértice de $K$ é adjacente a todos os vértices de $\mathrm{L}$, e portanto pode ser adicionado a $L$ para obtermos uma clique que intercepta $K$, assim como todas as cliques de $G$ interceptadas por $L$. Considere agora uma clique $K^{\prime}$ de $G$ que intercepta $C$ mas é disjunta de $L$. Segue, da definição de $L$, que tal clique não intercepta $K$. Precisamos considerar tal $K^{\prime}$ se ele contiver um vértice $a$ adjacente a algum vértice de $K$. Denote por $A$ o conjunto de tais vértices, i.e., vértices que são adjacentes a $K$ e pertencem a alguma clique que intercepta $C$ mas não $\mathrm{L}$. Afirmamos que cada $a \in A$ é adjacente a todos os vértices de $L$. Na verdade, se $b \in L$ não é adjacente a $a$, então existem vértices $c \in$ $C \backslash L$, e $s, t \in K$ (possivelmente $s=t$ ) tal que , $b, c, a, s, t, b$ é um ciclo sem corda. Similarmente, provamos que $A$ é uma clique, i.e., para quaisquer $a, a^{\prime} \in A, a$ e $a^{\prime}$ são adjacentes em $G$. Caso contrário, existem vértices $c, c^{\prime} \in C \backslash L$ (possivelmente $c=c^{\prime}$ ), e $s, s^{\prime} \in K$ (possivelmente $s=s^{\prime}$ ), tal que $a, c, c^{\prime}, a^{\prime}, s^{\prime}, s^{\prime} a$ é um ciclo sem corda. Logo, o conjunto $L \cup A$ induz uma clique, e Lema 1 garante que existe um vértice $u \in K$ adjacente a todos os vértices de $L \cup A$. Agora, a clique induzida por $L \cup A \cup u$ intercepta $K$ e, por definição de $A$, intercepta, também, todas as cliques interceptadas por $V$ que são adjacentes a $K$. 
Observe que o lema acima também é válido quando $C$ e $K$ não são disjuntos $\left(\operatorname{com} C^{\prime}=C\right)$.

Lema 3. Sejam $C_{1}, C_{2}, \ldots, C_{p}$ uma coleção de cliques duas a duas adjacentes num grafo cordal $G$. Então existe uma clique $C$ em $G$ que intercepta cada $C_{i}, i=$ $1,2, \ldots, p$.

Prova. O resultado segue facilmente quando $p \leq 2$. Assuma agora que $p>2$. Por indução, existe uma clique $C$ que intercepta $C_{i}$ para cada $i \in\{1, \ldots, p-1\}$. Se $C$ intercepta $C_{p}$, nada resta provar. Caso contrário, aplique o Lema 2 a $C$ e $C_{p}$.

Uma condição simples e necessária para que um grafo $G$ seja um grafo- $(k, l)$ é que ele não contenha $l+1$ cópias independentes de $K_{k+1}$. Na verdade, considere qualquer partição de $G$ em $k$ conjuntos independentes e $l$ cliques. Qualquer $K_{k+1}$ em $G$ teria de conter um vértice de uma das cliques na partição, e portanto, dentre as $(l+1)$ cópias dos $K_{k+1}$, duas delas deveriam interceptar a mesma clique, e logo possuiriam uma aresta ligando-as (o que significa que não eram independentes.) Verifica-se que para grafos cordais a condição acima é também suficiente. (Observe que a condição diz apenas que $(l+1) K_{k+1}$ não é um subgrafo induzido de $G$.) Derivamos este fato do seguinte resultado:

Teorema 1. Seja $G$ um grafo cordal, e seja $r \geq 1$ um inteiro. Então $f(G, r)=$ $g(G, r)$.

É claro que $f(G, r) \leq g(G, r)$ para qualquer $G$ e qualquer $r \geq 1$. Para provarmos a igualdade para grafos cordais, vamos proceder como segue.

Seja $G$ um grafo. Vamos definir $K^{r}(G)$ como o grafo com um vértice correspondendo a cada $K_{r}$ em $G$, e dois vértices adjacentes em $K^{r}(G)$ se, e somente se, os $K_{r}$ 's correspondentes não são independentes em $G$.

Lema 4. Para qualquer grafo $G, f(G, r)$ é o número de independência de $K^{r}(G)$. Para um grafo cordal $G, g(G, r)$ é o número de cobertura por cliques de $K^{r}(G)$.

Prova. A primeira sentença é óbvia. A segunda segue da observação de que podemos modificar qualquer cobertura por clique $C$ de $K^{r}(G)$, para construir uma coleção de (com o mesmo número) cliques que interceptam todos os $K_{r}$ 's de $G$, aplicando o Lema 3 a cada clique em $C$.

Lema 5. Se $G$ é cordal então $K^{r}(G)$ também o é.

Prova. Assuma que $W_{1}, W_{2}, \ldots, W_{q}, W_{1},(q \geq 4)$ é um ciclo sem cordas em $K^{r}(G)$. Isto significa que $W_{i}$ e $W_{j}$ são consecutivos no ciclo se, e somente se, os $K_{r}$ 's correspondentes em $G$ são adjacentes. Considere uma sequência $S$ de vértices de $G, S=\left(u_{1}, w_{1}, u_{2}, w_{2}, \ldots, u_{q}, w_{q}\right)$ (índices são tomados circularmente no intervalo $1 \ldots q)$. É claro que se $i$ e $j$ não são índices consecutivos, então os subconjuntos $\left\{u_{i}, w_{i}\right\}$ e $\left\{u_{j}, w_{j}\right\}$ não são adjacentes. Ocasionalmente, pode ocorrer $u_{i}=w_{i}$ ou $w_{i}=u_{i+1}$ para algum $i$, mas estas igualdades não podem ocorrer simultaneamente. 
Isto significa que todo vértice ocorrendo em $S$ aparece no máximo duas vezes, e duas ocorrências de um mesmo vértice usam, necessariamente, posições consecutivas em $S$. Estas observações mostram que podemos construir um ciclo $C_{0}$ em $G$ a partir de $S$ removendo ocorrências repetidas de vértices. Esta construção assegura que pelo menos um vértice de $\left\{u_{i}, w_{i}\right\}$ é escolhido para todo $i \in\{1, \ldots, q\}$. Logo, $C_{0}$ contém pelo menos 4 vértices. Além disso, $C_{0}$ é claramente um ciclo sem corda, uma contradição.

O Teorema 1 segue naturalmente dos Lemas 4 e 5 .

Prova do Teorema 1. Pelo Lema $5 K^{r}(G)$ é cordal, e logo perfeito. Portanto, o número de independência de $K^{r}(G)$ é igual a número de cobertura por cliques. O Lema 4 completa a prova.

A caracterização de grafos cordais- $(k, l)$ por subgrafos proibidos segue como uma consequência do teorema anterior.

Teorema 2. Um grafo cordal é um grafo- $(k, l)$ se, e somente se, não contém $(l+$ 1) $K_{k+1}$ como um subgrafo proibido.

Prova. Mostramos que um grafo cordal- $(k, l)$ não pode conter $l+1$ cópias independentes de $K_{k+1}$, i.e., não pode conter $(l+1) K_{k+1}$ como um subgrafo proibido. Por outro lado, o Teorema 1 implica que se um grafo cordal $G$ não contém $l+1$ cópias independentes de $K_{k+1}$, então $g(G, k+1) \leq l$. Isto significa que $G$ contém $l$ cliques cuja remoção deixa um subgrafo $G^{\prime}$ sem $K_{k+1}$. Como $G$ é perfeito, $G^{\prime}$ é $K$-colorível, portanto $G$ admite uma partição em $k$ conjuntos independentes e $l$ cliques.

\section{Os Algoritmos}

Como $k$ e $l$ são fixos, existe um número polinomial de subgrafos de $G$ com $(l+$ $1)(k+1)$ vértices, e portanto o Teorema 1 nos fornece um algoritmo polinomial para reconhecer grafos cordais- $(k, l)$. Existem, contudo, algoritmos mais eficientes. O algoritmo que apresentamos aqui possui complexidade $O(n(m+n))$ e nos fornece uma segunda prova para o Teorema 1.

Inicialmente revisamos o algoritmo padrão de coloração para grafos cordais. (Observe que testar a existência de uma $k$-coloração é equivalente a reconhecer grafos- $(k, 0)$.) Suponha que os vértices de $G$ obedecem a um esquema de eliminação perfeita (EEP) $1,2, \ldots, n[7]$. O Algoritmo Guloso Reverso procede na ordem $n, n-$ $1, \ldots, 1$, associando a cada vértice a menor cor disponível. Em outra palavras, para colorir G com as cores $s_{1}, s_{2}, \ldots$, colorimos o vértice $n$ com a cor $s_{1}$, e tendo colorido os vértices $n, n-1, \ldots, i+1$, colorimos o vértice $i$ com a cor $s_{d}$ onde $d$ é o menor índice tal que nenhum vizinho de $i$ dentre $i+1, i+2, \ldots, n$ foi colorido com a cor $s_{d}$. Observe que até este ponto, $i$ faz parte de um $K_{d}$, já que i tem um vizinho de cada uma das cores $s_{1}, s_{2}, \ldots, s_{d-1}$, que são mutualmente adjacentes. (Quaisquer 
dois vizinhos de $i$ dentre $i+1, i+2, \ldots, n$ são adjacentes, já que $1,2, \ldots, n$ é um EEP.) Segue que o Algoritmo Guloso Reverso fornece, em tempo $O(m+n)$, uma coloração mínima e uma clique máxima.

Estamos prontos para descrever nosso algoritmo. Seja $k \geq 0$ um inteiro. O algoritmo encontra o menor valor de $l$ (possivelmente $l=0$ ) para o qual $G$ é um grafo- $(k, l)$. Estaremos colorindo os vértices do grafo cordal de entrada $G$ com as cores $s_{1}, s_{2}, \ldots, s_{k}$ e $c_{1}, c_{2}, \ldots, c_{l}$. Através da execução do algoritmo, os vértices coloridos com a cor $s_{d}$ formarão um conjunto independente, e os vértices coloridos com a cor $c_{a}$ formarão uma clique. Denotaremos por $S_{i}$ o conjunto que consiste de i, e mais todos os vértices dentre $1,2, \ldots, i-1$ coloridos com $s_{1}, s_{2}, \ldots, s_{k}$.

O seguinte fato é facilmente obtido das definições, usando as propriedades do esquema de eliminação perfeita:

Lema 6. Se o vértice $i$ é adjacente ao primeiro vértice $j$ colorido com a cor $c_{a} e$ $j<i$, então $i$ é adjacente a todos os vértices $x<i$ coloridos com a cor $c_{a}$.

Este Lema nos permitirá facilmente testar se um dado vértice $i$ pode ser adicionado ou não a clique formada pelos vértices coloridos com a cor $c_{a}$.

\section{Algoritmo para Reconhecer Grafos Cordais- $(k, l)$}

Assuma que $G$ é um grafo cordal com um esquema eliminação perfeita $1,2, \ldots, n$.

- Colora o vértice 1 com a cor $s_{1}$

- Tendo colorido os vértices $1,2, \ldots, i-1$ sem usar a cor $c_{1}$ :

- remova as cores de $1,2, \ldots, i-1$ e colora $1,2, \ldots, i$ com as cores $s_{1}, s_{2}, \ldots$, $s_{k}$ (usando o Algoritmo Guloso Reverso), se possível, ou

- mantenha a coloração dos vértices $1,2, \ldots, i-1$, e colora $i$ com a cor $c_{1}$.

- Tendo colorido os vértices $1,2, \ldots, i-1$ e tendo usado as cores $c_{1}, c_{2}, \ldots, c_{a}$ :

- colora $i$ com a cor $c_{b}$, onde $b \leq a$ é o menor índice tal que $i$ é adjacente ao primeiro vértice colorido com a cor $c_{b}$, se tal índice existe, ou

- remova as cores dos vértices de $S_{i} \backslash i$ e colora $S_{i}$ com as cores $s_{1}, s_{2}, \ldots$, $s_{k}$ (usando o Algoritmo Guloso Reverso), se possível, ou

- mantenha a coloração de $1,2, \ldots, i-1$, e colora $i$ com a cor $c_{a+1}$.

No algoritmo acima, $l$ é o maior valor de $a$ tal que existe um vértice colorido $c_{a}$, ou $l=0$ se todos os vértices estão coloridos com as cores $s_{1}, s_{2}, \ldots, s_{k}$.

Como o funcionamento do algoritmo é dominado por no máximo $n$ aplicações do Algoritmo Guloso Reverso, segue que o tempo de execução é $O(n(m+n))$. 
Proposição 1. Se o algoritmo usa a cor $c_{p}$, então $G$ contém um $p K_{k+1}$ como subgrafo induzido.

Prova. Seja $v_{a}$ o primeiro vértice (no EEP) colorido com a cor $c_{a}$. O subgrafo de $G$ induzido por $S_{v_{a}-1}$ é $k$-colorível, mas nosso algoritmo achou impossível adicionar $v_{a}$ de forma que $S_{v_{a}}$ continuasse $k$-colorível. Logo existe um subgrafo isomorfo a $K_{k+1}$ contendo $v_{a}$ e alguns $k$ vértices de $S_{v_{a}-1}$. Resta apenas mostrar que os subgrafos $X_{1}, X_{2}, \ldots, X_{p}$ são independentes. Suponha que um vértice $x$ do subgrafo $X_{a}$ seja adjacente ou igual a um vértice $x^{\prime}$ de um subgrafo $X_{a^{\prime}}$. Assuma que $a<a^{\prime}$.

Se $x^{\prime} \leq x$, então devido ao fato de que $x^{\prime}$ é adjacente ou igual a $v_{a^{\prime}}$, concluímos que $x$ é adjacente ou igual a $v_{a^{\prime}}$. Agora $v_{a}$ e $v_{a^{\prime}}$ devem ser adjacentes, já que $x \leq v_{a}$ e $x \leq v_{a^{\prime}}$. Isto significa que $v_{a^{\prime}}$ é adjacente ao primeiro vértice colorido por $c_{a}$ contradizendo o fato de que nosso algoritmo pode colorir $v_{a^{\prime}} \operatorname{com}$ a cor $c_{a}$.

Se $x^{\prime}>x$, então $x$ é adjacente ou igual a $v_{a}$ por um argumento similar. Se $x^{\prime} \leq v_{a}$ então $v_{a^{\prime}}$ e $v_{a}$ devem ser adjacentes e $v_{a^{\prime}}$ deveria ter sido colorido com a cor $c_{a}$, como no caso anterior. Por outro lado, se $x^{\prime}>v_{a}$, então $x^{\prime}$ é adjacente ao primeiro vértice colorido com a cor $c_{a}$. Portanto, nosso algoritmo deveria ter colorido $x^{\prime}$ com a cor $c_{a}$, contradizendo o fato dele ter sido colorido com alguma cor $c_{d}$ ( no caso de $x$ ser um elemento de $S_{v_{d^{\prime}}-1}$ ) ou com a cor $c_{d^{\prime}}\left(\right.$ no caso $x^{\prime}=v_{d^{\prime}}$.)

Corolário 1. As seguintes afirmações são equivalentes:

1 - O algoritmo particiona $G$ em $k$ conjuntos independentes e $l$ cliques;

2 - O grafo $G$ é um grafo- $(k, l)$;

3 - O grafo $G$ não contém $(l+1) K_{k+1}$ como subgrafo induzido.

Prova: As implicações $1 \Rightarrow 2$ e $2 \Rightarrow 3$ são óbvias, e a Proposição 1 prova que $3 \Rightarrow 1$.

Observe que a equivalência entre 1 e 2 prova a corretude do algoritmo, enquanto que a equivalência entre 1 e 3 nos fornece uma segunda prova do Teorema 2 .

Encerramos esta seção observando que o algoritmo encontra, para qualquer $k \mathrm{e}$ para qualquer grafo cordal $G$, o menor valor $l$ tal que $G$ é um grafo- $(k, l)$.

\section{O Caso de um Conjunto Independente, Enfati- zando Grafos Split}

Quando $k=1$, podemos de alguma forma simplificar o algoritmo, já que não precisamos do Algoritmo Guloso Reverso para testar se um vértice pode ser adicionado a um conjunto independente, mantendo a propriedade de independência.

\section{Algoritmo para Reconhecer Grafos Cordais- $(1, l)$}

Assuma que $G$ é um grafo cordal com um EEP $1,2, \ldots, n$. 
- Colora o vértice $1 \mathrm{com}$ a cor $s_{1}$,

- e continue colorindo os vértices $i=2,3, \ldots$ com a cor $s_{1}$ enquanto for possível ( $i$ não tem aresta para $1,2, \ldots, i-1$ ),

- e então colora o primeiro $j$ que não pode ser colorido com a cor $c_{1}$.

- Tendo colorido os vértices $1,2, \ldots, i-1$ usando as cores $s_{1}, c_{1}, c_{2}, \ldots, c_{a}$,

- colora $i$ com a cor $c_{b}$, onde $b \leq a$ é o primeiro índice tal que $i$ é adjacente ao primeiro vértice colorido com a cor $c_{b}$, se tal índice existir, ou

- colora $i$ com a cor $s_{1}$ se $i$ é não adjacente a todos os vértices coloridos com a cor $s_{1}$, ou

- colora $i$ com a cor $c_{a+1}$.

É claro que este algoritmo pode ser implementado em tempo $O(m+n)$.

A situação é mais simples quando $k=l=1$, e neste caso não precisamos explicitamente assumir cordalidade. (Grafos split são automaticamente cordais.) Como grafo split são de grande interesse [7, 4], nós alteramos o algoritmo uma vez mais, de forma a reconhecer grafos split:

\section{Algoritmo para Reconhecer Grafos Split}

Seja $G$ um grafo qualquer.

- Encontre um EEP $1,2, \ldots, n$ de $G[7]$.

- Colora 1 com a cor $s$, e continue colorindo $i=2,3, \ldots$ com a cor $s$ enquanto for possível ( $i$ é não adjacente aos vértices já coloridos), então colora o próximo vértice $j$ com a cor $c$.

- Se todos os vértices $1,2, \ldots, i-1$ tiverem sido coloridos, e ambas as cores $s$ e $c$ já tiverem sido usadas, então colora $i$ com a cor $c$ se for adjacente ao primeiro vértice $j$ colorido com a cor $c$; caso contrário, colora $i$ com a cor $s$ se for não adjacente a todos os vértices já coloridos com a cor $s$.

Se o algoritmo falhar devido a não existência de um EEP, então o algoritmo dado em [7] exibe um subgrafo induzido isomorfo a um $C_{4}, C_{5}$, ou $C_{k}, k \geq 6$. Se ele falhar colorindo todos os vértices, então de acordo com a Proposição $1, G$ contém um subgrafo induzido isomorfo a um $2 K_{2}$. Apresentamos abaixo um pequena versão da prova, que será usada numa generalização mais abaixo.

Se, num certo momento, um vértice $i$ não pode ser colorido com a cor $s$ ou com a cor $c$, então ele é não adjacente ao primeiro vértice $j$ colorido com a cor $c$, e é 
adjacente a algum vértice $k$ que foi anteriormente colorido com a cor $s$. Afirmamos que $j$ e $k$ não podem ser adjacentes. Se $k<j$, segue das propriedades de EEP. Se $k>j$, então se $k$ fosse adjacente a $j$ o algoritmo teria colorido com a cor $c$.

O vértice $j$ foi colorido com a cor $c$, porque ele era adjacente a um vértice $l$ previamente colorido com a cor $s$. Como $l<j<i$, e $i, j$ são não adjacentes, $l$ deve ser não adjacente a $i$. Além disso, os vértices $k, l$ são não adjacentes, já que eles foram ambos coloridos com a cor $s$. Portanto, $i, j, k, l$ formam um subgrafo induzido isomorfo a um $2 K_{2}$ em $G$.

Como cada $C_{k}, k \geq 6$ também contém um subgrafo induzido isomorfo a um $2 K_{2}$, obtivemos a seguinte caracterização já conhecida de grafos split [7]:

Corolário 2. Um grafo $G$ é um grafo split se, e somente se, não contém $2 K_{2}, C_{4}$ ou $C_{5}$ como subgrafos induzidos.

\section{Referências}

[1] A. Brandstädt, Partitions of graphs into one or two independent sets and cliques, Discrete Mathematics, 152 (1996), 47-54.

[2] A. Brandstädt, The complexity of some problems related to graph 3colorability, Discrete Applied Mathematics, 89 (1998), 59-73.

[3] T. Feder, P. Hell, S. Klein, and R. Motwani, Complexity of graph partition problems, em "The 31st Annual ACM Symposium on Theory of Computing - STOC'99" ( F. W. Thatcher and R. E. Miller, eds.), pp. 464-472, Plenum Press, New York, 1999.

[4] S. Foldes and P. Hammer, Split Graphs, em " 8th Southeastern Conf. on Combinatorics, Graph Theory and Computing" (F. Hoffman et al., eds.), pp. 311-315, Louisiana State Univ., Baton Rouge, Louisiana.

[5] L. T. Nogueira, "Grafos Split e Grafos Split Generalizados", Tese de Mestrado, COPPE-Sistemas, Universidade Federal do Rio de Janeiro, Rio de Janeiro, RJ, Brazil, 1999.

[6] M. R. Garey, D. S. Johnson and L. Stockmeyer, Some simplified NP-complete graph problems, Theoretical Computer Science, 1 (1976), 237-267.

[7] M. C. Golumbic, "Algorithmic Graph Theory and Perfect Graphs", Academic Press, New York, 1980. 
